\title{
AVALIAÇÃO QUALITATIVA DE CARACTERÍSTICAS SENSORIAIS DE CLONES PRECOCES DO CAFÉ CONILON
}

\author{
Antônio Pereira Drumond Neto ${ }^{1}$ \\ Lucas Barbosa Laurindo ${ }^{2}$ \\ Tiago da Silva Machado ${ }^{3}$ \\ João Batista Pavesi Simâo ${ }^{4}$ \\ Samuel de Assis Silva ${ }^{5}$ \\ Julião Soares de Souza Lima ${ }^{6}$
}

Resumo: O objetivo do trabalho foi avaliar as notas das características sensoriais aroma, sabor e doçura do conjunto de clones precoces do café conilon Vitoria cultivado a altitude média de 520 m. Para realizar o trabalho, foram delimitados três talhões e colhidos 10 litros do fruto cereja por clone e as amostras foram divididas em: frutos desmucilado e em coco (natural). Após secagem em terreiro suspenso por $24 \mathrm{~h}$, utilizouse a estufa a 45 graus até as amostras atingirem teor de água de $12 \%$ bu. A análise sensorial foi feita pelo teste prova de xicara com três degustadores. Os clones precoces apresentaram diferenças significativas em relação ao processamento das amostras, com maior média para o aroma e a doçura na condição desmucilado. O clone 4 apresentou diferença significativamente para o aroma e a doçura comparativamente com os demais pelo teste de Tukey $(p<0,05)$. O sabor não apresentou diferença significativa no processamento das amostras entre os clones.

Palavras-chave: Prova de xicara; Café especia; Clones Vitoria.

\footnotetext{
${ }^{1}$ Agronomia/Universidade Federal do Espírito Santo, Brasil. E-mail: agrodrumond@gmail.com.

2 Agronomia/Universidade Federal do Espírito Santo, Brasil. E-mail: lucas.lb12@hotmail.com.

3 Agronomia/Universidade Federal do Espírito Santo, Brasil. E-mail: tiagosmachado_@hotmail.com.

${ }^{4}$ Agronomia/Instituto Federal do Espírito Santo, Brasil. E-mail: jbpavesi@ifes.edu.br.

${ }^{5}$ Agronomia/Universidade Federal do Espírito Santo, Brasil. E-mail: samuel.assilva@gmail.com.

${ }^{6}$ Engenheiro Agrícola/Universidade Federal do Espírito Santo, Brasil. E-mail: limajss@yahoo.com.br.
} 